\title{
Polarized resonant soft X-ray scattering measurements in polymer-grafted nanoparticles \\ Dean DeLongchamp
}

\author{
National Institute of Standards and Technology, Gaithersburg, United States of America;
}

\section{dean.delongchamp@nist.gov}

Orientation and conformation in nanoscale amorphous regions often dominate the properties of soft materials such as composites and semicrystalline polymers. Robust correlations between between structure in these amorphous regions and important properties are not well developed due to a lack of measurements with high spatial resolution and a sensitivity to molecular orientation. I will describe our approach to solving this issue using polarized resonant soft X-ray scattering (P-RSoXS), which combines principles of soft X-ray spectroscopy, small-angle scattering, real-space imaging, and molecular simulation to produce a molecular scale structure measurement for soft materials and complex fluids.

Because P-RSoXS is relatively new to the scattering community, I will first cover the basics of the measurement. The fundamental principles of P-RSoXS and near-edge X-ray absorption fine structure (NEXAFS) spectroscopy, the spectroscopic basis for P-RSoXS, will be reviewed. The P-RSoXS experiment will be discussed including sample preparation and constraints, which differ considerably from analogous scattering techniques such as conventional small-angle X-ray scattering (SAXS) and small angle neutron scattering (SANS). I will also cover approaches for including gases or liquids in the experiment, and describe available measurement facilities. Data collection best practices will be reviewed.

I will then describe polarized resonant soft X-ray scattering (P-RSoXS) measurements of model systems including polymer-grafted nanoparticles. Analysis will focus on quantitative extraction of orientation details from nanoscale glassy regions. This work is now accelerated by a powerful analysis framework using parallel computation across graphics processing units (GPUs) for the forwardsimulation of P-RSoXS patterns. In polymer-grafted nanoparticles, we can apply this framework to fit quantitative and detailed descriptions of amorphous chain orientation with $\approx 2 \mathrm{~nm}$ resolution.

Keywords: macromolecule, polymer, SAXS, XAFS, amorphous 